\title{
Inflammatory Pseudotumor of the Choroid Plexus
}

\author{
Mark Bramwit, Peter Kalina, and Marilou Rustia-Villa
}

\begin{abstract}
Summary: A case of inflammatory pseudotumor is described, in which CT revealed a hyperdense intraventricular lesion with a trapped temporal horn, and MR imaging showed decreased T1 and markedly hypointense T2 signal with homogeneously intense enhancement. Radiologically and grossly the tumor resembled a meningioma; however, histologically it was composed purely of inflammatory, nonneoplastic components. The lesion was resected with no evidence of recurrence at 5-month follow-up.
\end{abstract}

Index terms: Choroid plexus, inflammation; Brain neoplasms

Inflammatory pseudotumor is an uncommon lesion of unknown origin. Although the computed tomographic (CT) and magnetic resonance $(M R)$ imaging characteristics may simulate meningioma, pathologically these masses are composed of purely inflammatory, nonneoplastic components. We describe a patient with pseudotumor of the choroid plexus who presented with gradual clinical deterioration.

\section{Case Report}

A 63-year-old woman had a several-week history of frequent headaches and unsteady gait. She was brought to the emergency department after falling. A physical examination, she was slightly confused with a mild left-sided hemiparesis. Sensation was grossly intact. A CT scan (Fig 1A) was obtained on the day of admission, and an MR imaging study (Fig 1B-F) was performed 1 day after admission. The patient was treated with intravenous mannitol and steroids and improved clinically. She subsequently underwent ventricular shunting and excision of the mass. The preoperative diagnosis was an unusual meningioma.

During surgery, a smooth, slightly pink tumor was identified near the atrium of the right lateral ventricle. The tumor was well encapsulated and appeared to be attached to the choroid plexus. The tissue in the tumor felt gritty and was extremely tough and fibrous, suggestive of a meningioma. The attachment of the tumor along the medial and inferior wall of the temporal horn near the trigone was extremely adherent and difficult to dissect. A shunt cath- eter was passed from the lateral ventricle through the temporal horn, exiting over the convexity.

Histologically, the lesion consisted of fibrocartilagenous tissue with scattered inflammatory cells, entrapped reactive choroid plexus, and gliotic neuroparenchyma (Fig $1 G)$. No mitotic figures or nuclear pleomorphism were seen. Special stains for organisms, such as Ziehl-Neelsen stain for acid-fast bacilli and Gomori's methenamine silver stain for fungi were negative. No amyloid was identified on Congo red stain. By immunoperoxidase staining, the stromal cells were positive with S-100 protein; a few rare cells were immunoreactive with keratins $39,40,43,48,50,50.6$ $\mathrm{kd}$ (CAM5.2) and keratins 37,43,50 kd(CK) (CAM 5.2/ AE-1).

A 5-month follow-up contrast-enhanced CT scan (Fig $1 \mathrm{H})$ showed no evidence of recurrence. Eight months after surgery, the patient was asymptomatic with the exception of mild headaches. Physical examination revealed a leftsided superior quadrantanopia, which was attributed to postoperative changes in the region of the optic radiations. The patient was treated prophylactically with phenytoin for seizures.

\section{Discussion}

Inflammatory pseudotumor is a pathologic term used to describe a reactive, inflammatory, nonneoplastic proliferation of connective tissue. Inflammatory pseudotumor has been described most often in the lung, with other systemic sites somewhat less common. Primary central nervous system (CNS) involvement is rare (1). The pathologic mechanism for inflammatory pseudotumor remains obscure (2). In several reported cases, patients have had elevated levels of serum immunoglobulins, leading to the hypothesis that the inflammatory pseudotumors were the result of an exaggerated immunologic process (2). Inflammatory meningeal masses may represent a variant of meningioma in which an unusual immunologic response has been evoked (3). Those masses may show varying proportions of inflammatory and me-

Received April 23, 1996; accepted after revision November 8.

From the Department of Radiology, North Shore University Hospital, New York University School of Medicine, 300 Community Dr, Manhasset, NY 11030. Address reprint requests to Mark Bramwit, MD. 


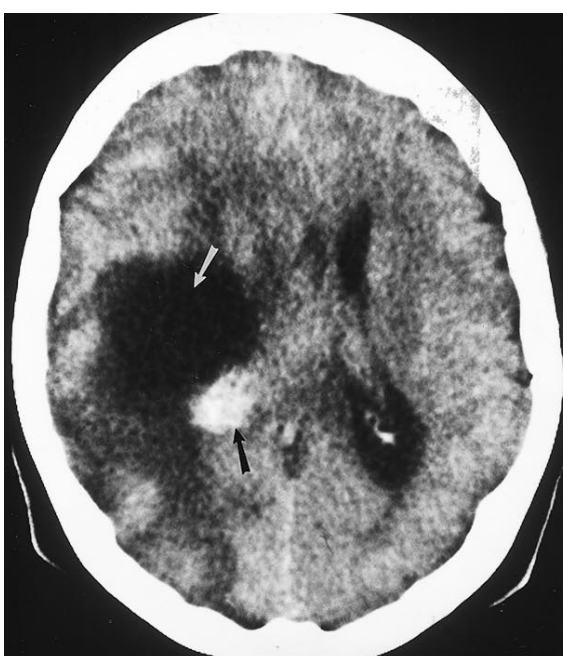

A

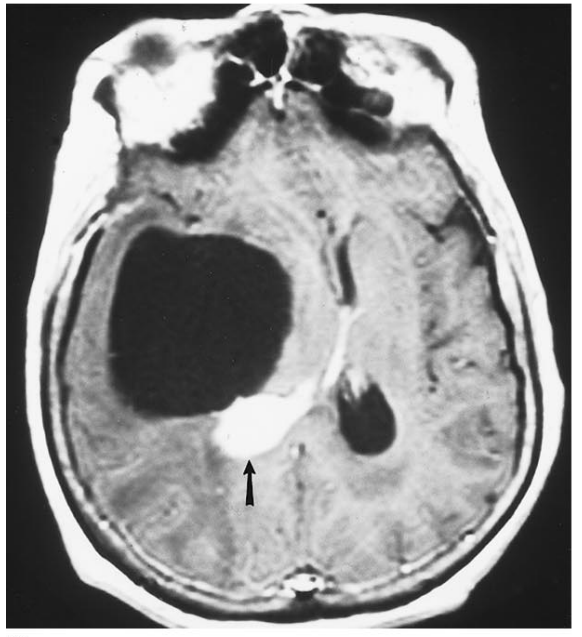

D

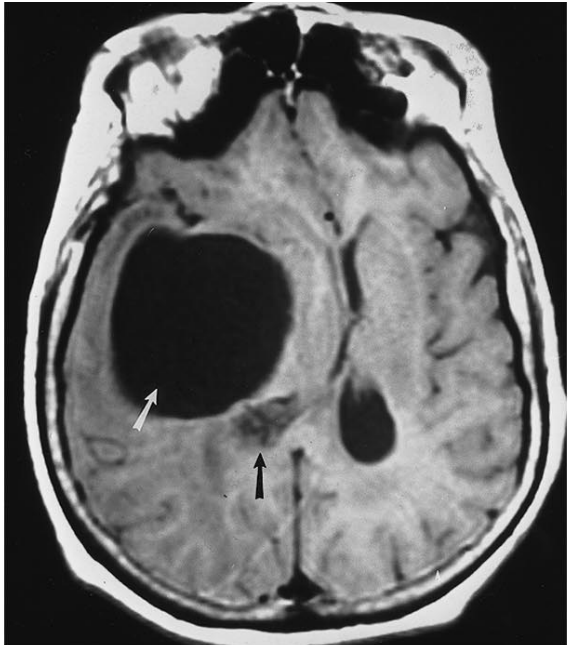

B

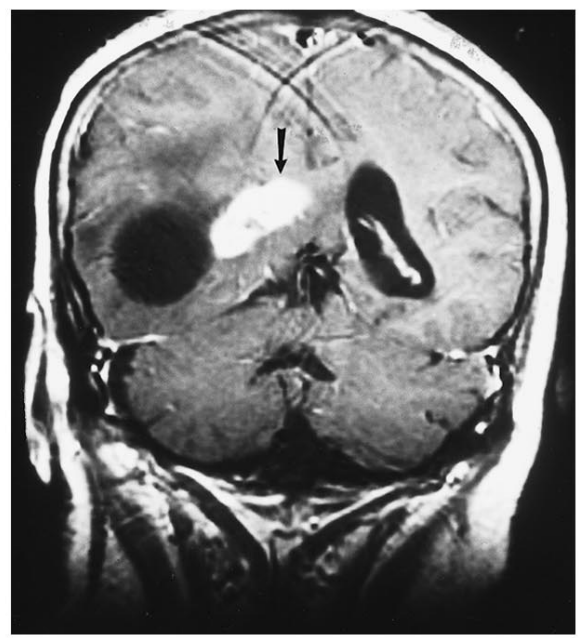

E

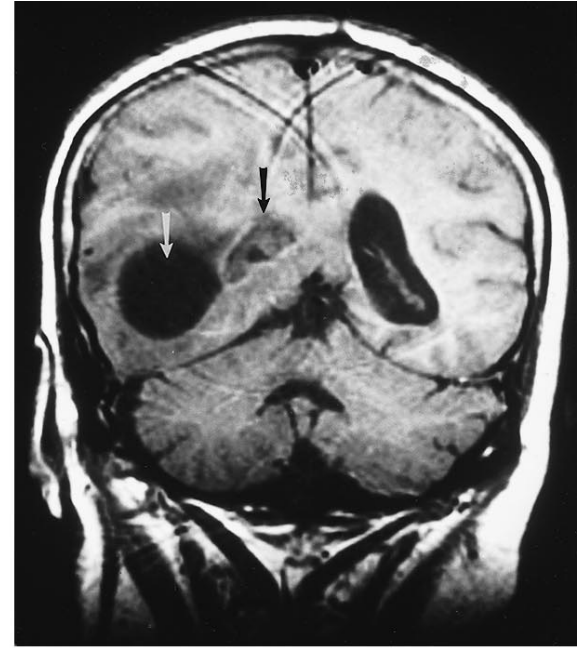

C

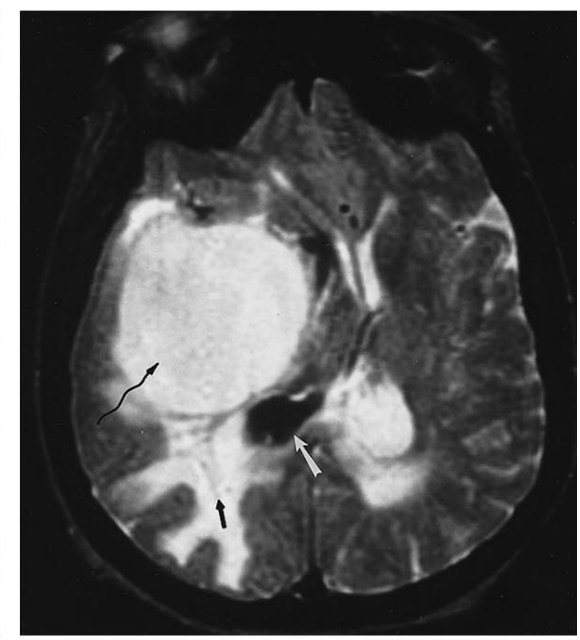

$\mathbf{F}$

Fig 1. A 63-year-old woman with inflammatory pseudotumor of the choroid plexus.

$A$, Noncontrast CT scan shows a hyperdense lesion in the atrium of the right lateral ventricle (black arrow) with adjacent vasogenic edema and mass effect. Note trapped right temporal horn (white arrow).

Axial $(B)$ and coronal $(C)$ T1 -weighted images (500/16/2 [repetition time/echo time/excitations]) show a well-defined, slightly hypointense lesion within the atrium of the right lateral ventricle (black arrow). Note the significant enlargement of the right temporal horn (white arrow).

Axial (D) and coronal (E) contrast-enhanced T1weighted images $(500 / 16 / 2)$ show homogeneous enhancement of the intraventricular mass (arrow).

$F$, Axial T2-weighted MR image (2500/80/1) shows significant hypointensity of the intraventricular mass (white arrow). The significantly enlarged right temporal horn (wavy black arrow) and vasogenic edema (straight black arrow) are again evident.

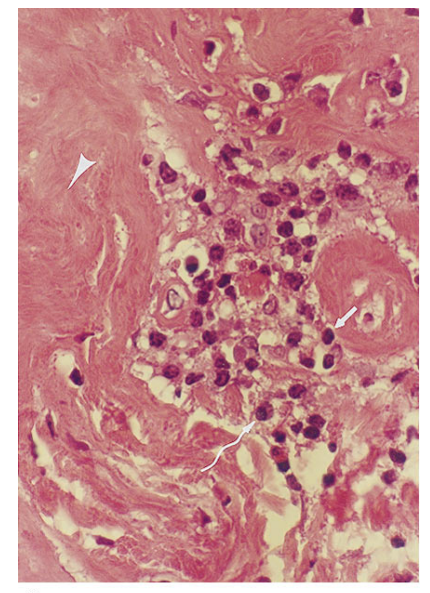

G

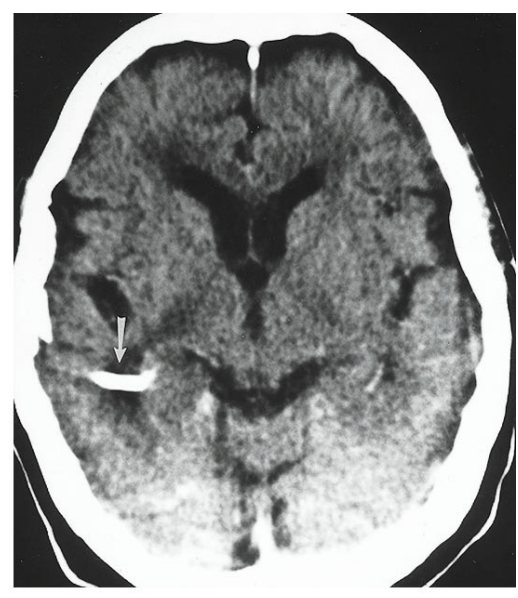

$\mathrm{H}$

G, Photomicrograph shows fibrocartilagenous tissue containing scattered inflammatory cells and entrapped reactive choroid plexus epithelium (arrowhead). The inflammatory cells are composed mainly of plasma cells (wavy arrow) and lymphocytes (straight arrow) (hematoxylin-eosin, original magnification $\times 40$ ).

$H$, Contrast-enhanced CT scan at 5-month follow-up shows no evidence of recurrence. A portion of the shunt catheter is seen in the region of the temporal lobe (arrow). 
ningothelial components. The spectrum of pathologic components varies from apparent meningiomas with a striking inflammatory component to exclusively inflammatory, nonneoplastic masses attached to the dura (3). Inflammatory pseudotumor of the CNS affects patients between the ages of 13 and 80 years. Unlike with meningiomas, the majority of these patients are under the age of 40 years (2). The lesions are seen to arise from different parts of the brain parenchyma and meninges without restriction to a particular zone (2).

In addition to inflammatory pseudotumor, the differential diagnostic considerations of a wellcircumscribed enhancing intraventricular mass in the lateral ventricle include meningioma, metastasis, xanthogranuloma, neurosarcoidosis, oligodendroglioma, astrocytoma, ependymoma, choroid plexus papilloma, and neurocytoma. Differentiating these entities from inflammatory pseudotumor can be facilitated by taking into account the patient's age as well as the location and imaging characteristics of the lesion. Metastases may be strongly suspected when there is a history of primary neoplasm. Xanthogranuloma, neurosarcoid, and low-grade astrocytomas are unlikely to hemorrhage. Ependymomas are more common in the fourth ventricle. Choroid plexus papilloma occurs in younger patients. Intraventricular neurocytoma and oligodendroglioma, although difficult to distinguish from each other, are frequently attached to the septum pellucidum.

Although the lesion in this case had a tumor- like gross presentation, histologic findings were nonneoplastic. The pronounced inflammatory reaction suggested an infectious process; however, organisms known to cause mass lesions in the choroid plexus, such as mycobacteria and various fungi, were not found. Another inflammatory process that may involve the choroid plexus is neurosarcoidosis; however, granulomata were not found. Xanthogranulomata was excluded owing to an absence of xanthoma cells. Collagen vascular diseases were excluded both clinically and histologically.

Given the radiologic and histologic characteristics, the lesion was classified as an inflammatory pseudotumor of the choroid plexus. Definitive treatment was complete surgical removal. This rare, benign entity should be considered in the differential diagnosis of intraventricular lesions.

\section{Acknowledgment}

We thank Marc K. Rosenblum for his assistance with this case.

\section{References}

1. Pimentel J, Corta A, Tavora L. Inflammatory pseudotumor of the choroid plexus. J Neurosurg 1993;79:939-941

2. Al-Sarraj S, Wasserberg J, Bartlett R, Bridges LR. Inflammatory pseudotumor of the central nervous system: clinicopathological study of one case and review of the literature. $B J$ Neurosurg 1995;9:57-66

3. Mirra SS, Tindall SC, Check IJ, Brynes RK, Moore WW. Inflammatory meningeal masses of unexplained origin. $J$ Neuropathol Exp Neurol 1983;4:453-468 